\title{
Respiratory Care Education and Clinical Practice in Mainland China
}

\author{
Jie Li MSc RRT RRT-NPS RRT-ACCS, Yuenan Ni MSc, Meilien Tu MSc, Ju Ni, \\ Huiqing Ge MSc, Yunfeng Shi, Zhong Ni, Rongchang Chen MD PhD, Rong Yao MD, \\ Zongan Liang MD; on behalf of the Respiratory Care Committee of Chinese Respiratory \\ Disease Society
}

\begin{abstract}
BACKGROUND: Compared with 10 years ago when our last survey was completed, the number of respiratory therapists (RTs) has increased markedly in mainland China. In addition, the education systems for RTs and the working environment have also changed. We aimed to describe the current status of respiratory care in mainland China. METHODS: A nationwide survey was initiated from August 15, 2016, to September 2, 2016, through network platforms. RESULTS: We obtained responses from $196 \mathrm{RTs}$, of whom, 30.6\% graduated from a bachelor's degree program, $25.5 \%$ graduated from an associate's degree program, and $43.9 \%$ were nurses who transitioned to be RTs through 6-month on-the-job training programs. Among the 3 groups, no significant differences existed in the basic job responsibility, such as mechanical ventilation and aerosol therapy; however, bachelor's degree RT graduates participated more in bronchoscopy assistance $(96 \%$ vs $78 \%$, $P=.002)$, extracorporeal membrane oxygenation management $(42 \%$ vs $25 \%, P=.02)$, and pulmonary ultrasound $(40 \%$ vs $15 \%, P<.001)$. There was no RT certification or licensure in mainland China at the time of the survey, so only $23 \%$ of bachelor's degree graduates and $42 \%$ of associate's degree graduates received a license through other professions. For the respondents' opinions on the obstacles of respiratory care profession development, the lack of licensure was a profound barrier for both degree graduates, whereas on-the-job training RTs deemed that insufficient recognition of the value of the respiratory care profession was the main obstacle. CONCLUSIONS: In mainland China, degree programs for students and on-the-job training for Experienced ICU nurses were 2 major ways to train RTs. The absence of credential and/or licensure and the lack of recognition of the value of an RT were deemed as the $\mathbf{2}$ key obstacles in the development of respiratory care profession. Key words: respiratory care practice; education; licensure. [Respir Care 2018;63(10):1239-1245. (C) 2018 Daedalus Enterprises]
\end{abstract}

\section{Introduction}

Respiratory therapists (RTs) have worked as independent professionals for more than 70 years in the United

\footnotetext{
$\mathrm{Ms} \mathrm{Li}$ is affiliated with the Division of Respiratory Care, Department of Cardiopulmonary Sciences, Rush University Medical Center, Chicago, Illinois. Ms Y Ni, Mr Z Ni, and Dr Liang are affiliated with the Department of Respiratory and Critical Care Medicine, West China School of Medicine and West China Hospital, Sichuan University, Chengdu, Sichuan, China. Ms Tu is affiliated with the Department of Respiratory Care, Chang Gung University of Science and Technology, Puzi City, Taiwan. Mr J Ni is affiliated with the Department of Respiratory Care, Zhengzhou Railway Vocational Technical College, Zhengzhou, Henan, China. Ms Ge is affiliated with the Department of Respiratory Care, Sir Run Run Show Hospital, Hangzhou, Zhejiang, China. Mr Shi is affiliated with the
}

States, and the effectiveness of RTs in mechanical ventilation, aerosol therapy, performing mini-bronchoalveolar lavage, and implementing respiratory care protocols to wean patients from mechanical ventilation has been shown. ${ }^{1-6}$

\footnotetext{
Department of Critical Care Medicine, West China School of Medicine and West China Hospital, Sichuan University, Chengdu, Sichuan, China. Dr Chen is affiliated with the Department of Respiratory and Critical Care Medicine, Guangzhou Institute of Respiratory Disease, Guangzhou, Guangdong, China. Dr Yao is affiliated with the Department of Emergency Medicine, West China School of Medicine and West China Hospital, Sichuan University, Chengdu, Sichuan, China.
}

Ms Li and Ms Y Ni contributed equally to this work.

The authors have disclosed no conflicts of interest 
However, in many countries, respiratory therapy has not been accepted as an independent profession in the medical field. The recognition of RTs is still in a transitional phase in some countries, such as mainland China, ${ }^{7}$ Germany, ${ }^{8}$ and the United Kingdom. A recent survey, which included 335 ICUs within 20 Asian countries indicated that only $40.6 \%$ of ICUs hired RTs. Ninety-nine ICUs were located in middle-income countries, and 85 of them were Chinese ICUs. Of those 99 ICUs, only $32.3 \%$ hired RTs. ${ }^{9}$ There are no specific data for Chinese ICUs, but this survey shows that the awareness of the value of RTs has increased because our previous study showed that only $9.7 \%$ ICUs (7/72) hired RTs 10 years ago in Beijing. ${ }^{7}$

In recent decades, intensive care medicine in China has developed rapidly ${ }^{10}$ and so has the respiratory care profession. From 2004 to 2009, there was only one program, which was a baccalaureate program, to train RTs in mainland China. From this program, only 10-20 students graduated each year, therefore, the respiratory care profession developed slowly. ${ }^{11}$ As suggested in our previous survey, ${ }^{7}$ different kinds of education programs have been established to expand the group of professional RTs as well as to meet the clinical needs. In the past 10 years, 2 associate's degree programs were established, and these programs have graduated $>100$ students. The baccalaureate program was built with the help of the American Association for Respiratory Care and Loma Linda University using similar curriculum to the American program. The associate's degree programs were established as a branch of a critical care nursing program; the curriculum includes nursing and respiratory care.

Based on the experience from Taiwan respiratory care development, $\mathrm{Li}^{13}$ started a 6-month on-the-job training program in 2009 to convert experienced ICU nurses to RTs. This program offers bedside instruction with weekly 4-8 $\mathrm{h}$ in-classroom teaching and monthly evaluation. This model was rapidly accepted by other hospitals. Currently, $>10$ hospitals use this model to provide onthe-job training. ${ }^{14}$ To simplify the description specifically in this article, RTs who graduated from a respira-

\footnotetext{
Ms Li, Ms Tu, Dr Liang, and Dr Yao conceived and designed the study. Ms Tu, Ms Y Ni, Mr Z. Ni, Ms Ge, and Mr Shi distributed the survey and collected data. Ms Y Ni and Ms Li analyzed and interpreted the data, and wrote the manuscript. Drs Liang and Chen provided critical revisions and approved the final version of the manuscript. Ms Y Ni and Ms Li are responsible for the entire manuscript.
}

Correspondence: Rong Yao, MD, Department of Emergency Medicine, West China School of Medicine and West China Hospital, Sichuan University, No. 37 Guo Xue Xiang, Wuhou District, Chengdu, Sichuan 610041, PR China. E-mail: 1037070596@qq.com.

DOI: $10.4187 /$ respcare. 06217

\section{QUICK LOOK}

\section{Current knowledge}

Respiratory therapists (RTs) contribute to the outcome of patients in the ICU and their effectiveness has been proven; however, currently, there is not a respiratory care profession in many countries. Respiratory therapy as an independent profession has started to make progress in many countries, such as mainland China, India, Argentina, and Saudi Arabia.

\section{What this paper contributes to our knowledge}

This study found that RTs were typically from 3 different educational programs in mainland China: bachelor's degree graduates, associate's degree graduates, and on-the-job training for experienced ICU nurses. We also compared RTs' job responsibilities as well as obstacles to their professional development. Licensure remains a priority for their professional development. Overall, the development of the respiratory care profession in mainland China might be used as a reference by other countries that have a similar medical environment and situation.

tory care bachelor's degree program are named bachelor's degree RTs. Similarly, respiratory care associate's degree program graduates are named associate's degree RTs, and nurses from on-the-job training are named as on-the-job training RTs. Because respiratory care practices have changed since 2007 , we sought to describe the current practice of RTs in mainland China and to compare the practice of RTs from different educational programs. In addition, we attempted to explore professional development obstacles that Chinese RTs encountered.

\section{Methods}

\section{Study Design}

This study was approved by the institutional ethical committee for clinical and biomedical research of West China Hospital (Sichuan, China). Because this was a prospective survey and no therapeutic intervention was included, written informed consent was waived. To develop the survey instrument, we used the background information obtained from our previous survey ${ }^{7}$ and informal interviews with experts in the fields of respiratory care and critical care medicine. A survey methodology was applied, including item generation and reduction, pretesting, sensibility, and test-retest reliability, to develop the questionnaire survey. 
This survey was sponsored by the Respiratory Care Committee of Chinese Respiratory Disease Society from August 15, 2016, to September 2, 2016, and was advertised on various social media and RT network platforms (eg, WeChat). An online survey tool (Tencent Questionnaire Tencent, Shenzhen, China) was used to perform the survey.

\section{Survey Items}

The self-administered, anonymous, descriptive survey had 51 items in 3 specific areas: (1) demographic characteristics, which included age, sex, department, job titles, the highest degree of education and the major for the highest degree, respiratory care education, the length of the career, certification, credentials, after-tax annual income; (2) daily job responsibilities: intubation, ventilator parameters setting, spontaneous breathing trial, extubation, monitoring, circuit management, ventilator management, oxygen therapy, aerosol therapy, suctioning, chest physiotherapy, arterial puncture, arterial blood gas analysis, pulmonary function test, bronchoscopy assistant, transportation of patients on mechanical ventilation, extracorporeal membrane oxygenation management, pulmonary ultrasound, nutrition management; (3) respondents' opinions toward the development of the respiratory care profession in their own department and in mainland China. The answers to the questions on the obstacles of RT development were listed as multiple choices (for example, the lack of the RT certification and credentials, insufficient educational programs, low income, and so forth) with a blank line for comments.

\section{Statistical Analysis}

Consecutive variables were reported as mean \pm SD or median (interquartile range), whereas categorical variables were reported as frequency and proportion. Answers to open questions were reviewed by 2 independent investigators (YN, JL). The Student $t$ test, Mann-Whitney test, and Kruskal-Wallis tests were used for comparing continuous variables; the chi-square test or Fisher exact test were used for comparing categorical variables. All analyses were performed by using SPSS 19.0 (SPSS Inc, Chicago, Illinois), and a 2 -sided $P$ value of $<.05$ was considered statistically significant.

\section{Results}

A total of 228 questionnaires were received; 27 were excluded because $>10 \%$ of the questions were not answered, and 5 were excluded because they were duplicate. Among the 196 effective respondents, 60 (30.6\%) were bachelor's degree RTs and 58 of them worked in hospitals.
In addition, 50 respondents $(25.5 \%)$ were associate's degree RTs, 48 of them worked in hospitals. Also, 86 (43.9\%) were on-the-job training RTs and 78 of them worked in hospitals. Not all questions were completely answered by all the respondents, so the number of replies varied by the questions. The results were analyzed based on RTs from different educational programs.

\section{Demographic Information}

Degree program RTs were younger than the on-the-job training RTs, and the years after graduation were shorter. Compared with the associate's degree RTs and on-the-job training RTs, the bachelor's degree RTs had longer years of working as an RT (Table 1). On-the-job trainees were more likely to have a license from other clinical professions compared with those who graduated from a degree program (32\% vs 79\%, $P<.001)$. Bachelor's degree RTs earned higher after-tax annual income than the associate's degree RTs and the on-the-job training RTs who had the same amount of working years.

\section{Daily Clinical Practice of Respiratory Care in ICU}

The major job responsibilities for RTs who worked in ICU are listed in Table 2: ventilator parameters setting and adjustment (99\%), mechanical ventilation monitoring (98\%), extubation (95\%), suctioning (94\%), spontaneous breathing trial (93\%), oxygen therapy (93\%), chest physical therapy (92\%), and aerosol therapy (85\%). There was no difference in these responsibilities among the RTs from different educational programs. However, compared with associate's degree RTs and on-the-job trainees, bachelor's degree RTs tended to participate more in bronchoscopy assistance (96\% vs $78 \%, P=.002$ ), transportation of patients who were on mechanical ventilation $(96 \%$ vs $82 \%$, $P=.01)$, extracorporeal membrane oxygenation management ( $42 \%$ vs $25 \%, P=.02$ ), and pulmonary ultrasound ( $40 \%$ vs $15 \%, P<.001)$. In contrast, bachelor's degree RTs participated less in ventilator circuit change and disinfection $(70 \%$ vs $96 \%, P<.001)$ and ventilator maintenance ( $85 \%$ vs $97 \%, P=.003$ ) (Table 3 ).

\section{Opinions on the Development of Respiratory Care}

Of all the respondents, 94\% (185/196) stated that it was necessary to have an official certification or license for RTs. With regard to the major obstacles in the development of the respiratory care profession, RTs from different educational programs had diverse answers. RTs who graduated from degree programs deemed that the lack of RT certification and licensure $(75 \%, 83 / 110)$ was the major obstacle, whereas on-the-job trainees believed that insufficient recognition of the value of the 
Table 1. Characteristics of the RTs From Different Educational System

\begin{tabular}{|c|c|c|c|}
\hline Basic Characteristics & $\begin{array}{l}\text { RTs Graduated From } \\
\text { Bachelor's Degree } \\
\text { Program }(n=60)\end{array}$ & $\begin{array}{l}\text { RTs Graduated From } \\
\text { Associate's Degree } \\
\text { Program }(n=50)\end{array}$ & $\begin{array}{l}\text { RTs, On-the-Job } \\
\text { Training }(n=86)\end{array}$ \\
\hline Age, mean $\pm \mathrm{SD}, \mathrm{y}$ & $27 \pm 4$ & $25 \pm 2$ & $30 \pm 5$ \\
\hline Men, $n(\%)$ & $25(42)$ & $16(32)$ & $44(51)$ \\
\hline \multicolumn{4}{|l|}{ Department, $n(\%)$} \\
\hline ICU & $48(80)$ & $35(70)$ & $56(65)$ \\
\hline Pulmonary general unit & $5(8.3)$ & 0 & 0 \\
\hline Respiratory care & $5(8)$ & $12(24)$ & $19(22)$ \\
\hline Respiratory care product company & $1(2)$ & $2(4)$ & $4(5)$ \\
\hline Missing data & $1(2)$ & 0 & $4(5)$ \\
\hline \multicolumn{4}{|l|}{ Highest education, $n(\%)$} \\
\hline Master's degree & $3(5)$ & $1(2)$ & $3(3)$ \\
\hline Bachelor's degree & $57(95)$ & $10(30)$ & $70(81)$ \\
\hline Associate's degree & 0 & $39(78)$ & $13(15)$ \\
\hline Years since graduation from the highest degree, mean $\pm \mathrm{SD}$ & $3.87 \pm 3.34$ & $2.05 \pm 2.63$ & $5.26 \pm 3.23$ \\
\hline \multicolumn{4}{|l|}{ License, $n(\%)$} \\
\hline Physician & $3(5)$ & 0 & 0 \\
\hline Nurse & 0 & $7(14)$ & $67(78)$ \\
\hline Physical therapists & $11(18)$ & $14(28)$ & $1(1)$ \\
\hline No license & $46(77)$ & $29(58)$ & $18(21)$ \\
\hline \multicolumn{4}{|l|}{ Job rank, $n(\%)$} \\
\hline No job rank & $17(28)$ & $25(50)$ & $4(5)$ \\
\hline Level 1 & $31(52)$ & $25(50)$ & $47(55)$ \\
\hline Level 2 & $12(20)$ & $0(0)$ & $32(37)$ \\
\hline Level 3 & 0 & $0(0)$ & $3(3)$ \\
\hline \multicolumn{4}{|l|}{ Training institutes } \\
\hline University medical center in mainland China & 0 & 0 & 62 \\
\hline Taiwan hospital/school & 0 & 0 & 4 \\
\hline American hospital/school & 0 & 0 & 1 \\
\hline Other & 0 & 0 & 7 \\
\hline Years of working as an $\mathrm{RT}$, mean $\pm \mathrm{SD}$ & $4.34 \pm 3.48$ & $1.98 \pm 1.01$ & $2.89 \pm 3.09$ \\
\hline \multicolumn{4}{|l|}{ After-tax annual income (in US dollars), $n(\%)$} \\
\hline Not provided & $4(7)$ & $1(2)$ & $3(3)$ \\
\hline$\leq \$ 6,000$ & $13(22)$ & $23(46)$ & $6(7)$ \\
\hline$\$ 6,000-\$ 8,500$ & $3(5)$ & $17(34)$ & $23(27)$ \\
\hline$\$ 8,500-\$ 15,000$ & $16(27)$ & $9(18)$ & $34(40)$ \\
\hline$\$ 15,000-\$ 22,000$ & $19(32)$ & $0(0)$ & $15(17)$ \\
\hline$\$ 22,000-\$ 28,500$ & $4(7)$ & $0(0)$ & $2(2)$ \\
\hline$>\$ 28,500$ & $1(2)$ & $0(0)$ & $3(3)$ \\
\hline $\mathrm{RT}=$ respiratory therapist & & & \\
\hline
\end{tabular}

respiratory care profession $(87 \%)$ as well as a lack of certification and licensure (84\%) were the 2 major barriers in the development of the respiratory care profession. Regarding the major limitation in the development of their own groups or departments, associate's degree RTs chose insufficient training (52\%) as their major barrier, and on-the-job training RTs considered that shortage of RT staff (66\%) and insufficient training (51\%) were 2 major obstacles, whereas none of these factors were chosen by $>50 \%$ of the bachelor's degree RTs (Table 3).

\section{Discussion}

To our knowledge, this is the first study from mainland China conducted to compare the practice of RTs from different educational programs. No matter what type of training the RTs received, the respiratory care service they offered was standardized, with the utilization of evidence-based protocols. For example, $93 \%$ of RTs in our present study stated that they performed spontaneous breathing trials for patients in their ICUs. However, in our 2 previous surveys, only $40.9 \%^{15}$ and $57.8 \%^{7}$ of the participants stated that they per- 


\section{ResPiratory CARE IN CHINA}

Table 2. Comparison on Job Responsibility of RTs From Different Educational Programs

\begin{tabular}{|c|c|c|c|}
\hline Job Responsibility & $\begin{array}{c}\text { RTs Graduated From } \\
\text { Bachelor's Degree Program, } \\
n(\%)(n=53)\end{array}$ & $\begin{array}{c}\text { RTs Graduated From } \\
\text { Associate's Degree Program, } \\
n(\%)(n=48)\end{array}$ & $\begin{array}{l}\text { RTs, On-the-Job } \\
\text { Training, } n(\%) \\
\quad(n=78)\end{array}$ \\
\hline Intubation & $28(53)$ & $24(50)$ & $35(45)$ \\
\hline Ventilator setting adjustment & $53(100)$ & $47(98)$ & $78(100)$ \\
\hline Spontaneous breathing trial & $53(100)$ & $44(92)$ & $70(90)$ \\
\hline Extubation & $52(98)$ & $45(94)$ & $73(94)$ \\
\hline Mechanical ventilation monitoring & $53(100)$ & $48(100)$ & $74(95)$ \\
\hline Circuit change and disinfection* $* \dagger$ & $37(70)$ & $47(98)$ & $74(95)$ \\
\hline Ventilator maintenance ${ }^{* \dagger}$ & $45(85)$ & $47(98)$ & $75(96)$ \\
\hline Oxygen therapy & $48(91)$ & $44(92)$ & $75(96)$ \\
\hline Aerosol therapy & $44(83)$ & $39(81)$ & $70(90)$ \\
\hline Suctioning & $50(94)$ & $44(92)$ & $75(96)$ \\
\hline Chest physical therapy & $48(91)$ & $42(88)$ & $74(95)$ \\
\hline Arterial puncture* & $34(64)$ & $20(42)$ & $56(72)$ \\
\hline Arterial blood gas analysis & $36(68)$ & $32(67)$ & $55(71)$ \\
\hline Pulmonary function test & $21(40)$ & $22(46)$ & $26(33)$ \\
\hline Bronchoscopy assistant $* \dagger$ & $51(96)$ & $37(77)$ & $61(78)$ \\
\hline Transportation of ventilated patients $\dagger$ & $51(96)$ & $37(77)$ & $66(85)$ \\
\hline ECMO management* & $22(42)$ & $7(15)$ & $24(31)$ \\
\hline Pulmonary ultrasound $* \dagger$ & $21(40)$ & $4(8)$ & $15(19)$ \\
\hline Nutrition assessment & $11(21)$ & $6(13)$ & $24(31)$ \\
\hline Other & $30(57)$ & $16(33)$ & $30(38)$ \\
\hline
\end{tabular}

Table 3. Obstacles of RT Development for RTs From Different Educational Programs

\begin{tabular}{|c|c|c|c|}
\hline Obstacle & $\begin{array}{c}\text { RTs Graduated From } \\
\text { Bachelor's Degree Program, } \\
n(\%)(n=60)\end{array}$ & $\begin{array}{l}\text { RTs Graduated From } \\
\text { Associate's Degree } \\
\text { Program, } n(\%)(n=50)\end{array}$ & $\begin{array}{l}\text { RTs, On-the-Job } \\
\text { Training, } n(\%) \\
\quad(n=86)\end{array}$ \\
\hline Necessity of RT certification/licensure & $54(90)$ & $48(96)$ & $83(97)$ \\
\hline \multicolumn{4}{|c|}{ Obstacles of RT development in my department } \\
\hline Shortage of RT professionals & $19(32)$ & $20(40)$ & $57(66)$ \\
\hline Insufficient training for RT & $17(28)$ & $26(52)$ & $44(51)$ \\
\hline Insufficient recognition of RTs' value & $12(20)$ & $16(32)$ & $39(45)$ \\
\hline Economic factors & $17(28)$ & $13(26)$ & $37(43)$ \\
\hline No limitation & $21(35)$ & $6(12)$ & $5(6)$ \\
\hline Not provided & $3(5)$ & $1(2)$ & $3(3)$ \\
\hline \multicolumn{4}{|c|}{ Obstacles of RT development in mainland China } \\
\hline Scope of practice is limited & $9(15)$ & $10(20)$ & $32(37)$ \\
\hline Insufficient recognition on RTs' value & $9(15)$ & $14(28)$ & $75(87)$ \\
\hline Insufficient RT education programs & $10(17)$ & $10(20)$ & $37(43)$ \\
\hline Lack of certification and license & $47(78)$ & $36(72)$ & $72(84)$ \\
\hline Economic factors & $2(3)$ & $5(10)$ & $29(34)$ \\
\hline
\end{tabular}

formed spontaneous breathing trials. It needs to be noted that the majority of the participants in the 2 studies were ICU physicians and nurses who shared the respiratory care work due to the lack of RTs.
Because the medical environment varies from country to country, the development of the respiratory care profession is also quite varied. ${ }^{16,17}$ There is a high demand for RTs in mainland China ${ }^{7,14}$ and in other countries, such as 


\section{ResPiratory CARE IN ChinA}

Saudi Arabia and India, ${ }^{16,18}$ due to the rapid progress of intensive care medicine. ${ }^{9,16}$ Formal degree programs are not plentiful enough to supply professionals and expand the group of available RTs. In some developed countries, for example, Saudi Arabia, 15\% of RTs were trained in the United States. ${ }^{18}$ However, the expense of training abroad is massive, and this model of training may not be feasible in many developing countries. Also, building degree programs is a complicated, slow, costly, and labor-intensive process. Therefore, the on-the-job training for experienced ICU nurses is a more realistic and reasonable way to produce RTs because nurses already have medical background knowledge and ICU working experience. This type of educational program was used in the early phase of respiratory care development in Taiwan and Saudi Arabia. ${ }^{16,18}$

However, no study has been completed to prove its effectiveness. We found that the job responsibilities in ventilator management, airway management, and bronchial hygiene treatment were similar in the 3 groups. In our previous national survey within 320 ICUs, nurses in the majority of ICUs took the responsibilities of oxygen therapy, aerosol therapy, and suctioning. ${ }^{15}$ They were allowed to set or adjust ventilator settings in $<2 \%$ of ICUs. ${ }^{15}$ In contrast, our study demonstrated that almost all of the on-the-job training RTs who were ICU nurses before attending the training program took the same responsibilities on ventilator management as RTs who graduated from degree programs.

Even though the majority of the job responsibilities for RTs from different educational programs were the same, some differences still exist: bachelor's degree RTs were more involved in advanced or critical procedures, such as extracorporeal membrane oxygenation management and pulmonary ultrasound. This may be explained because the bachelor's degree RTs are more likely to obtain a job in teaching hospitals, which have more access to advanced techniques and more learning opportunities. In contrast, some of the basic procedures were performed less by bachelor's degree RTs, such as ventilator circuit changes and disinfection, ventilator maintenance, which includes, for example, self-test, and flow or oxygen sensor calibration. The bachelor's degree RTs also received a higher salary than the other 2 RT groups.

Due to the development of technology as well as the progression of intensive care medicine, the demands for RTs' skills and competency will increase. ${ }^{19}$ Thus, RTs in China will need more advanced training to satisfy the increasing demands. A minimum academic criterion to enter into the respiratory care profession is necessary in the future, when the RT population expands and a formal profession is built. Currently, respiratory care is still at its early phase of development in mainland China. The professional value of RTs still needs to be recognized. In the development of the profession, RT certification and licensure is a major and urgent obstacle, which also exists in other countries, for example, Saudi Arabia. ${ }^{17,18}$ Relying on the certification by the National Board for Respiratory Care, which is an American agency, is not a realistic solution. Being credentialed by a Chinese organization may be more feasible. Thus, this task needs to be listed as a priority by our Respiratory Care Committee.

Respiratory care is still uniquely a North American career in many aspects today. The progress of the respiratory care profession in mainland China might be referenced by other countries that are interested in developing this profession. Moreover, the comparison of the practice of RTs from different educational programs may also offer the opportunity for RTs to consider their career paths. According to Kacmarek and Walsh, ${ }^{19}$ the autonomy is explicitly linked to the educational level. One limitation to our study was that we did not investigate the quality of the work performed by RTs trained in different educational programs. In addition, we did not compare the outcome of the patients treated by different groups of RTs. The other limitation was the inability to survey all the graduates from the 3 educational programs. It might be possible that some of the graduates did not work as RTs any more, especially the associate's degree graduates because their program was a combined program with nursing and respiratory care, and the graduates can work as nurses as well. Plus, there are many hospitals that provide training for ICU nurses, and it is difficult to contact all their trainees.

\section{Conclusions}

The respiratory care profession in mainland China expanded rapidly in the past 10 years due to the establishment of the associate's degree programs and on-the-job training programs. Nevertheless, a bachelor's degree education is still highly demanded. Currently, the absence of licensure is the major obstacle in the development of the respiratory care profession.

\section{ACKNOWLEDGMENTS}

We thank J. Brady Scott MSc RRT RRT-ACCS FAARC and Tyler Weiss MSc RRT RRT-ACCS AE-C for reviewing the manuscript.

\section{REFERENCES}

1. Dailey RT, Malinowski T, Baugher M, Rowley DD. Impact of a Respiratory Therapy Assess-and-Treat Protocol on Adult Cardiothoracic ICU Readmissions. Respir Care 2017;62(5):517-523.

2. Kollef MH, Shapiro SD, Clinkscale D, Cracchiolo L, Clayton D, Wilner R, Hossin L. The effect of respiratory therapist-initiated treatment protocols on patient outcomes and resource utilization. Chest 2000;117(2):467-475.

3. Kallam A, Meyerink K, Modrykamien AM. Physician-ordered aerosol therapy versus respiratory therapist-driven aerosol protocol: the effect on resource utilization. Respir Care 2013;58(3):431-437.

4. Colice GL, Carnathan B, Sung J, Paramore LC. A respiratory therapistdirected protocol for managing inpatients with asthma and COPD incorporating a long-acting bronchodilator. J Asthma 2005;42(1):29-34. 


\section{Respiratory CARE IN ChinA}

5. Harbrecht BG, Delgado E, Tuttle RP, Cohen-Melamed MH, Saul MI, Valenta CA. Improved outcomes with routine respiratory therapist evaluation of non-intensive-care-unit surgery patients. Respir Care 2009;54(7):861-867.

6. Stoller JK. The effectiveness of respiratory care protocols. Respir Care 2004;49(7):761-765.

7. Li J, Zhan QY, Liang ZA, Tu ML, Sun B, Yao XL, et al. Respiratory care practices and requirements for respiratory therapists in Beijing intensive care units. Respir Care 2012;57(3):370-376.

8. Karg O, Bubulj C, Esche B, Geiseler J, Bonnet R, Mäder I. [The respiratory therapist.] Pneumologie 2008;62(11):685-689.

9. Arabi YM, Phua J, Koh Y, Du B, Faruq MO, Nishimura M, et al.; Asian Critical Care Clinical Trials Group. Structure, Organization, and Delivery of Critical Care in Asian ICUs. Crit Care Med 2016; 44(10): e940-e948.

10. Du B, Xi X, Chen D, Peng J; China Critical Care Clinical Trial Group (CCCCTG). Clinical review: critical care medicine in mainland China. Crit Care 2010;14(1):206.

11. The respiratory care division at the west China school of medicine at Sichuan university. http://www.irccouncil.org/newsite/news/2016/ 03/west_china.cfm. Accessed May 24, 2018.
12. Introduction of Taiwan society for respiratory therapy. http:// www.tsrt.org.tw/Introducition_e.asp?id=28. Accessed May 24, 2018.

13. Li J. Expanding the RT group in mainland China. AARC Times 2010;12:48-49.

14. Rowley DD, Sharkey R. Respiratory care expansion in China. AARC Times 2016;12:25-27.

15. Li J, Zhan QY, Liang ZA, Du ML, Dai HP, Sun B, et al. A questionnaire survey on the current practices of respiratory care in intensive care unit in 30 provinces. Zhongguo Wei Zhong Bing Ji Jiu Yi Xue 2009;21(4):211-214.

16. Esquinas A, Maynard D, Zuffo S, Chu CC, Garza HL, Olguin G, et al. Respiratory care around the world. International Council for Respiratory Care: Reports from the ICRC board of governors. AARC Times 2007;44-55.

17. Khosravi A, Rezaee R, Sabetian G. Benchmarking, a new method for developing an educational curriculum for respiratory therapy courses in medical schools. J Contemp Med Educ 2014;2(2):97-101.

18. Alotaibi G. Status of respiratory care profession in Saudi Arabia: A national survey. Ann Thorac Med 2015;10(1):55-60.

19. Kacmarek RM, Walsh BK. The Respiratory Therapy Profession Is at a Crossroads. Respir Care 2017;62(3):384-386. 